PROCEEDINGS OF THE

AMERICAN MATHEMATICAL SOCIETY

Volume 139, Number 12, December 2011, Pages 4369-4375

S 0002-9939(2011)10944-4

Article electronically published on April 21, 2011

\title{
A NOTE ON COMPOSITION OPERATORS ACTING ON HOLOMORPHIC SOBOLEV SPACES
}

\author{
BOO RIM CHOE, HYUNGWOON KOO, AND WAYNE SMITH
}

(Communicated by Richard Rochberg)

\begin{abstract}
A holomorphic self-map $\varphi$ of the unit disk is constructed such that the composition operator induced by $\varphi$ is bounded on the Hardy-Sobolev space $H_{2}^{1}$ of order 2 as well as on the ordinary holomorphic Lipschitz space Lip 1 but unbounded on the Zygmund class $\Lambda_{1}$. Among these three function spaces we have embedding relations $H_{2}^{1} \subset \operatorname{Lip}_{1} \subset \Lambda_{1}$. So, the main points here are that our construction provides a composition operator which is bounded on smaller spaces, but not on a larger space and that all the function spaces involved are standard ones.
\end{abstract}

\section{INTRODUCTION}

Let $\mathbf{D}:=\{z \in \mathbf{C}:|z|<1\}$ be the unit disk in the complex plane $\mathbf{C}$ and $\varphi$ be a holomorphic self-map of $\mathbf{D}$, i.e., $\varphi(\mathbf{D}) \subset \mathbf{D}$. The composition operator $C_{\varphi}$ induced by such a $\varphi$ is the linear operator on the space of all holomorphic functions on $\mathbf{D}$ defined by $C_{\varphi}(f)=f \circ \varphi$. Books of Cowen and MacCluer [5] and Shapiro [12] are good references for various aspects of the basic theory of composition operators.

It is typically (but not always; see below) the case that when a composition operator is bounded on a smaller space, it is also bounded on a larger space. To illustrate such a phenomenon for holomorphic Sobolev spaces, we recall the holomorphic version of the well-known Sobolev embedding theorem: For $\alpha \geq-1, s>0$ and $0<p<\infty$, we have

$$
s p>\alpha+2 \Longrightarrow A_{\alpha, s}^{p} \subset \Lambda_{s-(\alpha+2) / p} ;
$$

see [2, Section 5]. Here $A_{\alpha, s}^{p}$ is the $\alpha$-weighted Bergman-Sobolev space on $\mathbf{D}$ consisting of all holomorphic functions with fractional derivatives of order $s$ belonging to the well-known $\alpha$-weighted Bergman space $A_{\alpha}^{p}$, and $\Lambda_{a}$ denotes the holomorphic Lipschitz space of order $a>0$. Here, $A_{-1, s}^{p}$ is to be interpreted as the HardySobolev space, denoted by $H_{s}^{p}$. Explicit definitions will be given in Section 2.

It is known that if $C_{\varphi}$ is bounded on $A_{\alpha, s}^{p}$ and $\alpha+2<s p<\alpha+2+p$, then $C_{\varphi}$ is also bounded on $\Lambda_{s-(\alpha+2) / p}$; see [3, Theorem 3.2]. The same implication was later

Received by the editors October 18, 2010.

2010 Mathematics Subject Classification. Primary 47B33; Secondary 30C35, 46E35.

Key words and phrases. Composition operator, holomorphic Sobolev spaces, Zygmund class.

The first two authors were supported by the Korea Research Foundation Grant funded by the Korean Government (KRF-2008-314-C00012).

The third author wishes to acknowledge the hospitality of Korea University, where this research was carried out.

(C)2011 American Mathematical Society Reverts to public domain 28 years from publication 
shown to hold for $s p>\alpha+2+p$, leaving only those parameters with $s p=\alpha+2+p$ missing; see [4, Theorems 1.3 and 1.4]. The following natural question concerning the missing critical case was posed in [4, Problem 3.9]:

Let $s p=\alpha+2+p$ and suppose that $C_{\varphi}$ is bounded on $A_{\alpha, s}^{p}$. Does it follow that $C_{\varphi}$ is bounded on $\Lambda_{1}$ ?

Here we present the unexpected result that the answer to this question is "No" in general.

Theorem 1.1. There exists a holomorphic self-map $\varphi$ of $\mathbf{D}$ such that the composition operator $C_{\varphi}$ is bounded on $H_{2}^{1}$ and bounded on $\operatorname{Lip}_{1}$, but not bounded on $\Lambda_{1}$

As mentioned above, other examples are known of composition operators bounded on a smaller space, but not bounded on a larger space. One can be found in [5, Example 3.4], with the Hardy space $H^{2}$ as the smaller space and a certain weighted sequence space as the larger space. While that example is simple, with $\varphi(z)=z^{2}$ being the inducing function for the composition operator, the example given here is the only one we know that involves only "standard" function spaces. The tradeoff is that the construction of the inducing map $\varphi$ in our example is rather subtle.

In Section 2 we review the holomorphic Sobolev spaces and Lipschitz spaces and provide some background on the hyperbolic metric that will be used in our construction of the holomorphic self-map $\varphi$ of $\mathbf{D}$ to prove Theorem 1.1. That construction will be carried out in Section 3.

Notation. In the rest of the paper we use the notation $A \lesssim B$ or $B \gtrsim A$ for nonnegative quantities $A$ and $B$ to mean $A \leq C B$ for some inessential constant $C>0$. Similarly, we use the notation $A \approx B$ if both $A \lesssim B$ and $B \lesssim A$ hold. We will also use the notation $A(x) \sim B(x)$ as $x \rightarrow x_{0}$ for positive quantities $A(x)$ and $B(x)$ to mean that $A(x)$ and $B(x)$ are asymptotic; i.e., $A(x) B(x)^{-1} \rightarrow 1$ as $x \rightarrow x_{0}$.

\section{BACKGROUND}

2.1. Holomorphic Lipschitz spaces. For $0<\epsilon<1$, we let $\Lambda_{\epsilon}$ denote the space of all functions $f$ holomorphic on $\mathbf{D}$ and continuous on $\overline{\mathbf{D}}$ such that

$$
\|f\|_{\Lambda_{\epsilon}}:=|f(0)|+\sup \frac{|f(z)-f(w)|}{|z-w|^{\epsilon}}<\infty
$$

where the supremum is taken over all $z, w \in \mathbf{D}, z \neq w$. For $\epsilon=1$, we use $\Lambda_{1}$ to denote the Zygmund class of all functions $f$ holomorphic on $\mathbf{D}$ and continuous on $\overline{\mathbf{D}}$ such that

$$
\|f\|_{\Lambda_{1}}:=|f(0)|+\left|f^{\prime}(0)\right|+\sup \frac{\left|f\left(e^{i(\theta+h)}\right)+f\left(e^{i(\theta-h)}\right)-2 f\left(e^{i \theta}\right)\right|}{h}<\infty,
$$

where the supremum is taken over all $e^{i \theta} \in \partial \mathbf{D}$ and $h>0$. Equipped with norms $\|\cdot\|_{\Lambda_{\epsilon}}$, these spaces $\Lambda_{\epsilon}$ are all Banach spaces.

We remark in passing that the classes $\Lambda_{\epsilon}, 0<\epsilon \leq 1$, admit well-known characterizations in terms of the growth rate of the first derivative for $0<\epsilon<1$ and of the second derivative for $\epsilon=1$; see, for example, [5] or [6]. Also, in conjunction with the embedding property (1.1), we remark (although not needed in this paper) that 
the notion of holomorphic Lipschitz spaces extends to arbitrary order in a standard way. Namely, given a positive integer $n$ and $0<\epsilon \leq 1$, the space $\Lambda_{n+\epsilon}$ consists of all functions $f$ holomorphic on $\mathbf{D}$ and $n$-times continuously differentiable on $\overline{\mathbf{D}}$ such that $f^{(n)} \in \Lambda_{\epsilon}$ (with norm appropriately adjusted).

A characterization of when a composition operator is bounded on the Zygmund class has been known by various authors; see [9, Theorem 1], [8, Theorem 2.1] or [4, Theorem 2.2].

Theorem 2.1 (9], 8], 4]). Let $\varphi$ be a holomorphic self-map of $\mathbf{D}$. Then $C_{\varphi}$ is bounded on $\Lambda_{1}$ if and only if

$$
\sup _{z \in \mathbf{D}} \frac{\left|\varphi^{\prime}(z)\right|^{2}(1-|z|)}{1-|\varphi(z)|}<\infty
$$

and

$$
\sup _{z \in \mathbf{D}}\left|\varphi^{\prime \prime}(z)\right|(1-|z|) \log \left(\frac{2}{1-|\varphi(z)|}\right)<\infty .
$$

Let $\operatorname{Lip}_{1}$ be the space of all functions corresponding to $\epsilon=1$ in (2.1). It is well known and elementary that $f \in \operatorname{Lip}_{1}$ if and only if $f^{\prime} \in H^{\infty}$, the space of all bounded holomorphic functions on $\mathbf{D}$, with $\|f\|_{\text {Lip }_{1}} \approx|f(0)|+\left\|f^{\prime}\right\|_{\infty}$. Also elementary is that $C_{\varphi}$ is bounded on $\operatorname{Lip}_{1}$ if and only if $\varphi^{\prime} \in H^{\infty}$. Of course, $\operatorname{Lip}_{1} \subset \Lambda_{1}$.

2.2. Holomorphic Sobolev spaces. For $0<p<\infty$ and $\alpha>-1$, the weighted Bergman space $A_{\alpha}^{p}$ is the space of all functions $f$ holomorphic on $\mathbf{D}$ for which

$$
\|f\|_{A_{\alpha}^{p}}:=\left\{\int_{\mathbf{D}}|f(z)|^{p} d A_{\alpha}(z)\right\}^{1 / p}<\infty .
$$

Here $d A_{\alpha}$ denotes the weighted measure $d A_{\alpha}(z)=\left(1-|z|^{2}\right)^{\alpha} d A(z)$, where $d A$ is the area measure on $\mathbf{D}$. Also, the Hardy space $H^{p}$ is the space of all $f$ holomorphic on $\mathbf{D}$ for which

$$
\|f\|_{H^{p}}:=\sup _{0<r<1}\left\{\int_{0}^{2 \pi}\left|f\left(r e^{i \theta}\right)\right|^{p} d \theta\right\}^{1 / p}<\infty .
$$

We will often use the notation $A_{-1}^{p}=H^{p}$, which allows unified statements.

For $s \in \mathbf{R}$ and $f(z)=\sum_{n=0}^{\infty} a_{n} z^{n}$ holomorphic on $\mathbf{D}$, the $s$-fractional derivative $\mathcal{R}^{s} f$ of $f$ is defined by

$$
\mathcal{R}^{s} f(z)=\sum_{n=0}^{\infty}(1+n)^{s} a_{n} z^{n}, \quad z \in \mathbf{D}
$$

see [2]. For $\alpha \geq-1, s \geq 0$, and $0<p<\infty$, the holomorphic Sobolev space $A_{\alpha, s}^{p}$ is defined to be the space of all functions $f$ holomorphic on $\mathbf{D}$ such that $\mathcal{R}^{s} f \in A_{\alpha}^{p}$ with "norm"

$$
\|f\|_{A_{\alpha, s}^{p}}:=\left\|\mathcal{R}^{s} f\right\|_{A_{\alpha}^{p}}
$$

As mentioned above, we often write $H_{s}^{p}=A_{-1, s}^{p}$. In case $1 \leq p<\infty$, which is the case for the space $H_{2}^{1}$ to appear in our example, the spaces $A_{\alpha, s}^{p}$ are all Banach spaces. In conjunction with the question stated in the introduction, we clarify that the term "boundedness" even for $0<p<1$ refers to boundedness with respect to the above norm. 
When $s$ is an integer, the Sobolev norm can be computed using ordinary derivatives. That is, when $s$ is a positive integer,

$$
\|f\|_{A_{\alpha, s}^{p}} \approx \sum_{k=0}^{s-1}\left|f^{(k)}(0)\right|+\left\|f^{(s)}\right\|_{A_{\alpha}^{p}} ;
$$

see, for example, 44. Thus, for example, $f \in H_{2}^{1}$ if and only if $f^{\prime} \in H_{1}^{1}$ if and only if $f^{\prime \prime} \in H^{1}$. Meanwhile, a classical theorem of Privalov ([6. Theorem 3.11]) states that functions in $H_{1}^{1}$ continuously extend up to $\overline{\mathbf{D}}$ and their boundary functions are absolutely continuous on $\partial \mathbf{D}$. In particular, $H_{2}^{1} \subset \operatorname{Lip}_{1}$.

Composition operators acting on holomorphic Sobolev spaces were studied by the current authors in [3] and [4]. One result that will be used below is that $C_{\varphi}$ is bounded on $H_{2}^{1}$ if and only if $\varphi \in H_{2}^{1}$; see [13] or [4, Theorem 1.4].

2.3. The hyperbolic metric. The hyperbolic metric will play an important role in our construction. On the unit disk the hyperbolic metric $\rho_{\mathbf{D}}$ is defined by (see [1, p. 2])

$$
\rho_{\mathbf{D}}\left(z_{1}, z_{2}\right)=\inf \left\{\int_{\gamma} \frac{2|d z|}{1-|z|^{2}}: \gamma \text { is an arc in } \mathbf{D} \text { from } z_{1} \text { to } z_{2}\right\} .
$$

For example, $\rho_{\mathbf{D}}(0, z)=\log \frac{1+|z|}{1-|z|}$ is computed by integrating along the radius from 0 to $z$. This metric is invariant under conformal self-maps of the disk and therefore transfers to a natural conformally invariant metric on any simply connected domain $\Omega \subsetneq \mathbf{C}$. If $\psi: \mathbf{D} \rightarrow \Omega$ is any conformal map, the hyperbolic distance on $\Omega$ is given by $\rho_{\Omega}\left(w_{1}, w_{2}\right)=\rho_{\mathbf{D}}\left(z_{1}, z_{2}\right)$, where $w_{j}=\psi\left(z_{j}\right)$ for $j=1,2$. Since

$$
\frac{2|d z|}{1-|z|^{2}}=\frac{2|d w|}{\left|\psi^{\prime}(z)\right|\left(1-|z|^{2}\right)}, \quad \text { where } w=\psi(z),
$$

$\rho_{\Omega}$ can be computed by integrating the function

$$
h_{\Omega}(w)=\frac{2}{\left|\psi^{\prime}(z)\right|\left(1-|z|^{2}\right)}, \quad w=\psi(z),
$$

over $\operatorname{arcs}$ in $\Omega$. The following lemma is immediate from this observation.

Lemma 2.2. If $\gamma$ is a crosscut of a simply connected domain $\Omega$ that separates the points $a$ and $b$ in $\Omega$, then

$$
\rho_{\Omega}(a, b) \geq \inf _{c \in \gamma} \rho_{\Omega}(c, b) .
$$

Let $\delta_{\Omega}(z)$ denote the distance from a point $z$ in a region $\Omega$ to the boundary of $\Omega$. It is an easy consequence of the Koebe Distortion Theorem that if $\psi$ is a conformal map of $\mathbf{D}$ onto $\Omega$, then

$$
\frac{1}{4}\left(1-|z|^{2}\right)\left|\psi^{\prime}(z)\right| \leq \delta_{\Omega}(\psi(z)) \leq\left(1-|z|^{2}\right)\left|\psi^{\prime}(z)\right| ;
$$

see [11, Corollary 1.4]. Along with (2.3), this leads to the very useful Euclidean lower bound for the hyperbolic distance given in the following lemma.

Lemma 2.3 (12, Distance Lemma, p. 157]). If $a$ and $b$ are points of a simply connected domain $\Omega$, then

$$
\rho_{\Omega}(a, b) \geq \frac{1}{2} \log \left(1+\frac{|a-b|}{\min \left\{\delta_{\Omega}(a), \delta_{\Omega}(b)\right\}}\right) .
$$




\section{ProOF}

This section is devoted to the proof of Theorem 1.1

Proof of Theorem 1.1. For each integer $n \geq 1$ define

$$
\Gamma_{n}:=\left\{z \in \mathbf{D}:|1-z|=2^{-n} \text { and }|\operatorname{Im} z| \geq \epsilon_{n}\right\},
$$

where $\epsilon_{n}>0$ is sufficiently small and will be chosen later so that $\epsilon_{n}$ tends to 0 as $n \rightarrow \infty$. Now set

$$
\Omega:=\mathbf{D} \backslash \bigcup_{n=1}^{\infty} \Gamma_{n}
$$

and let $\psi$ be the Riemann map of $\mathbf{D}$ onto $\Omega$ such that $\psi(0)=0$ and $\psi^{\prime}(0)>0$. Since $\Omega$ is symmetric about the real axis, the uniqueness of the Riemann map implies that $\psi$ is real on and only on $(-1,1)$. Moreover, $\psi$ maps the upper (lower, resp.) half of $\mathbf{D}$ onto the upper (lower, resp.) half of $\Omega$. Observe that the boundary of $\Omega$ has finite one-dimensional Hausdorff measure, and so $\psi^{\prime} \in H^{1}$ by [10. Theorem 10.11] (see also [7, Exercise VI.1]) and thus that $\psi$ extends to be continuous on the closed disk $\overline{\mathbf{D}}$ by a theorem of Privalov ( 6 , Theorem 3.11]) mentioned before. Moreover, we have $\psi(1)=1$ (and $\psi(-1)=-1$ ), because $\psi^{\prime}(0)>0$.

For each integer $n \geq 1$, set

$$
x_{n}=1-\frac{1}{2}\left(\frac{1}{2^{n}}+\frac{1}{2^{n+1}}\right)
$$

so that $x_{n}$ is midway between $\Gamma_{n}$ and $\Gamma_{n+1}$, and $\delta_{n}:=\delta_{\Omega}\left(x_{n}\right) \approx 2^{-n}$ for all $n$. Then, with $r_{n} \in(-1,1)$ defined so that $\psi\left(r_{n}\right)=x_{n}$, from (2.4) we have

$$
\left|\psi^{\prime}\left(r_{n}\right)\right|\left(1-r_{n}^{2}\right) \approx \delta_{n} \approx 2^{-n}
$$

for all $n$. It is important to note that this estimate is independent of the choice of $\left\{\epsilon_{n}\right\}$. Next, we show below that this sequence can be chosen to make the distances $\rho_{n}:=\rho_{\mathbf{D}}\left(0, r_{n}\right)=\rho_{\Omega}\left(0, x_{n}\right)$ as large as needed.

For each integer $n \geq 1$, let

$$
\gamma_{n}:=\left\{z \in \mathbf{D}:|1-z|=2^{-n} \text { and }|\operatorname{Im} z|<\epsilon_{n}\right\} \subset \Omega .
$$

Note that $\gamma_{n}$ is a crosscut of $\Omega$ that separates the points 0 and $x_{n}$. To begin with, choose $\epsilon_{n}>0$ such that

$$
\sup _{w \in \gamma_{n}} \delta_{\Omega}(w) \leq 2 \epsilon_{n} \leq \delta_{\Omega}\left(x_{n}\right),
$$

which holds for all $\epsilon_{n}$ sufficiently small (when $n$ is fixed). Accordingly, applying Lemmas 2.2 and 2.3. we see from (3.2) that

$$
\begin{aligned}
\rho_{n}=\rho_{\Omega}\left(0, x_{n}\right) & \geq \inf _{w \in \gamma_{n}} \rho_{\Omega}\left(w, x_{n}\right) \\
& \geq \frac{1}{2} \inf _{w \in \gamma_{n}} \log \left(1+\frac{\left|w-x_{n}\right|}{2 \epsilon_{n}}\right) .
\end{aligned}
$$

Thus, since

$$
\inf _{w \in \gamma_{n}}\left|w-x_{n}\right|=x_{n}-\left(1-\frac{1}{2^{n}}\right)=\frac{1}{2^{n+2}}
$$

we obtain

$$
\rho_{n} \geq \frac{1}{2} \log \left(1+\frac{1}{\epsilon_{n} 2^{n+3}}\right)
$$


for each $n \geq 1$. This shows that we can make $\rho_{n}$ as large as we want by choosing $\epsilon_{n}>0$ sufficiently small. We now make this choice to insure that $2^{-n} \rho_{n} \geq n$, in addition to (3.2), for each $n \geq 1$. Then, from (3.1),

$$
\left|\psi^{\prime}\left(r_{n}\right)\right|\left(1-r_{n}^{2}\right) \log \frac{1+r_{n}}{1-r_{n}}=\left|\psi^{\prime}\left(r_{n}\right)\right|\left(1-r_{n}^{2}\right) \rho_{n} \gtrsim n
$$

for all $n$.

Let $g$ be the holomorphic function on $\mathbf{D}$ determined by the conditions that $g(0)=0$ and $g^{\prime}=\psi+1$. Note that $g$ is continuously differentiable on $\overline{\mathbf{D}}$. Since $g^{\prime}>0$ on $(-1,1)$, we have

$$
g\left(x_{1}\right)<g\left(x_{2}\right), \quad-1<x_{1}<x_{2}<1 .
$$

Also, since $\psi$ maps the upper half of $\mathbf{D}$ onto the upper half of $\Omega$, we have $\operatorname{Im} \psi(x+i t)>0$ for $x+i t \in \mathbf{D}$ (of course $x$ and $t$ denote real numbers) with $t>0$ and thus

$$
\operatorname{Re}[g(x+i y)-g(x)]=\operatorname{Re} \int_{0}^{y} \psi(x+i t) i d t<0
$$

for all $x+i y \in \mathbf{D}$ with $y>0$. The same inequality remains valid for $y<0$, because $\psi$ maps the lower half of $\mathbf{D}$ onto the lower half of $\Omega$. It follows from (3.4) and (3.5) that

$$
\operatorname{Re} g(z)<g(1)=\int_{0}^{1} \psi(x) d x+1, \quad z \in \mathbf{D} .
$$

Setting $s:=g(1)$, we thus see that $g$ maps $\mathbf{D}$ into the half-plane $\Pi_{s}:=\{z \in \mathbf{C}$ : $\operatorname{Re} z<s\}$. Note $s>0$.

Let $\sigma$ denote the Möbius map from the half-plane $\Pi_{s}$ onto $\mathbf{D}$ determined by the conditions $\sigma(0)=0, \sigma(s)=1$ and $\sigma(\infty)=-1$, i.e.,

$$
\sigma(z)=\frac{z}{2 s-z}, \quad z \in \Pi_{s}
$$

Now let $\varphi$ be the holomorphic self-map of $\mathbf{D}$ defined by $\varphi=\sigma \circ g$, and note that $\varphi(0)=0$ and $\varphi(1)=1$. Also, note that $\varphi$ is real on $(-1,1)$.

Note that $\left|\psi^{\prime}\left(r_{n}\right)\right| \rightarrow \infty$ as $n \rightarrow \infty$ by (3.3). Also, $r_{n} \rightarrow 1$ as $n \rightarrow \infty$. Thus, since $g^{\prime}=\psi+1$, and $\sigma$ is holomorphic at $s$ with $\sigma^{\prime}(s)=2 / s$, we compute

$$
\begin{aligned}
\left|\varphi^{\prime \prime}\left(r_{n}\right)\right|\left(1-r_{n}^{2}\right) & =\left|\psi^{\prime}\left(r_{n}\right) \sigma^{\prime}\left(g\left(r_{n}\right)\right)+\left(\psi\left(r_{n}\right)+1\right)^{2} \sigma^{\prime \prime}\left(g\left(r_{n}\right)\right)\right|\left(1-r_{n}^{2}\right) \\
& \sim \frac{2}{s}\left|\psi^{\prime}\left(r_{n}\right)\right|\left(1-r_{n}^{2}\right)
\end{aligned}
$$

as $n \rightarrow \infty$.

Since $\varphi\left(r_{n}\right)$ is real and converges to 1 as $n \rightarrow \infty$, we see that $\varphi\left(r_{n}\right)$ is positive for all large $n$. Thus, to each of such large $n$ corresponds $t_{n} \in\left(r_{n}, 1\right)$ such that

$$
1-\left|\varphi\left(r_{n}\right)\right|=\varphi(1)-\varphi\left(r_{n}\right)=\varphi^{\prime}\left(t_{n}\right)\left(1-r_{n}\right)
$$

by the Mean Value Theorem. Note that $g^{\prime}(1)=\psi(1)+1=2>0$; one may also use (3.6) and the Hopf Lemma to see the positivity of $g^{\prime}(1)$, which we need below. Now, since

$$
\varphi^{\prime}(t)=\sigma^{\prime}(g(t)) g^{\prime}(t) \rightarrow \sigma^{\prime}(s) g^{\prime}(1)=\frac{4}{s}>0
$$

as $t \rightarrow 1$, we see that

$$
1-\left|\varphi\left(r_{n}\right)\right| \sim \frac{4}{s}\left(1-r_{n}\right)
$$


as $n \rightarrow \infty$ and thus

$$
\log \left(\frac{1+r_{n}}{1-r_{n}}\right) \approx \log \left(\frac{2}{1-\left|\varphi\left(r_{n}\right)\right|}\right)
$$

for all large $n$. Combining this estimate with (3.7) and (3.3), we obtain

$$
\left|\varphi^{\prime \prime}\left(r_{n}\right)\right|\left(1-r_{n}^{2}\right) \log \frac{2}{1-\left|\varphi\left(r_{n}\right)\right|} \gtrsim n
$$

for all large $n$, which shows that Condition (2.2) is violated for $\varphi$. So, Theorem 2.1 shows that $C_{\varphi}$ is not bounded on $\Lambda_{1}$, as required.

It remains to check that $C_{\varphi}$ is bounded on $H_{2}^{1}$ and on $\operatorname{Lip}_{1}$. First note that $\sigma^{\prime}(z)=2 s(2 s-z)^{-2}$ and $\sigma^{\prime \prime}(z)=4 s(2 s-z)^{-3}$ are both bounded on $\Pi_{s}$, which contains the range of $g$. So, $\sigma^{\prime}(g)$ and $\sigma^{\prime \prime}(g)$ are both bounded on $\mathbf{D}$. Hence $\varphi^{\prime}=(\psi+1) \sigma^{\prime}(g) \in H^{\infty}$, and so $C_{\varphi}$ is bounded on $\operatorname{Lip}_{1}$; see the comment after Theorem 2.1. Also, since $\psi^{\prime} \in H^{1}$, we have $\varphi^{\prime \prime}=(\psi+1)^{2} \sigma^{\prime \prime}(g)+\psi^{\prime} \sigma^{\prime}(g) \in H^{1}$, which tells us that $C_{\varphi}$ is bounded on $H_{2}^{1}$; see the remark at the end of subsection 2.2. This completes the proof.

\section{REFERENCES}

[1] L. V. Ahlfors, Conformal Invariants, McGraw-Hill, 1973. MR0357743 (50:10211)

[2] F. Beatrous and J. Burbea, Holomorphic Sobolev spaces on the ball, Dissertationes Mathematicae, 276 (1989), 60 pp. MR1010151 (90k:32010)

[3] B. R. Choe, H. Koo and W. Smith, Composition operators acting on holomorphic Sobolev spaces, Trans. Amer. Math. Soc. 355(2003), 2829-2855. MR1975402 (2004e:47032)

[4] B. R. Choe, H. Koo and W. Smith, Composition operators on small spaces, Integr. Equ. Oper. Theory 56(2006), 357-380. MR2270842(2007h:47042)

[5] C. C. Cowen and B. D. MacCluer, Composition Operators on Spaces of Analytic Functions, CRC Press, 1995. MR1397026 (97i:47056)

[6] P. Duren, Theory of $H^{p}$ Spaces, Academic Press, 1970. MR0268655 (42:3552)

[7] J. B. Garnett and D. E. Marshall, Harmonic Measure, Cambridge Univ. Press, 2005. MR:2150803 (2006g:31002)

[8] S. Ohno, K. Stroethoff and R. Zhao, Weighted composition operators between Bloch-type spaces, Rocky Mountain J. Math. 33(2003), 191-215. MR1994487 (2004d:47058)

[9] S. Ohno and R. Zhao, Weighted composition operators on the Bloch space, Bull. Austral. Math. Soc. 63(2001), 177-185. MR.1823706 (2002d:47040)

[10] Ch. Pommerenke, Univalent Functions, Vandenhoeck and Ruprecht, 1975. MR0507768 $(58: 22526)$

[11] Ch. Pommerenke, Boundary Behavior of Conformal Maps, Springer-Verlag, 1992. MR1217706 (95b:30008)

[12] J. H. Shapiro, Composition Operators and Classical Function Theory, Springer-Verlag, 1993. MR1237406 (94k:47049)

[13] N. Zorboska, Composition operators on $S_{a}$ spaces, Indiana Univ. Math. J. 39(1990), 847-857. MR 1078741 (91k:47070)

Department of Mathematics, Korea University, Seoul 136-713, Republic of Korea

E-mail address: cbr@korea.ac.kr

Department of Mathematics, Korea University, Seoul 136-713, Republic of Korea

E-mail address: koohw@korea.ac.kr

Department of Mathematics, University of Hawait, Honolulu, Hawail 96822

E-mail address: wayne@math.hawaii.edu 